\title{
Fabrication, Performances and Aging of Flexible Gas
}

\section{Sensor Platforms}

Beatriz Medina-Rodriguez ${ }^{1 *}, 2$, Francisco Ramos ${ }^{1}$, Giovanni Vescio ${ }^{2}$, Xavier Arrese ${ }^{2}$, Aïda Varea ${ }^{2}$ and Albert Cirera $^{2}$

1. Advanced Materials Dept., FAE-Francisco Albero S. A. U., L'Hospitalet de Llobregat 08908, Spain

2. Department of Electronics, University de Barcelona, Barcelona 08028, Spain

\begin{abstract}
Flexible electronics are attracting much interest in gas sensor field on on-site monitoring applications due to their wear ability, lightweight and low-cost production. These performances are fully accomplished by silver-based platforms, but silver corrosion represents the main drawback for the integrity of the devices. In this work, self-heating sensor platforms fabricated by screen- and inkjet-printing techniques have been developed. The reliability of both types of sensors has been tested by long-term lifetime characterization and aging tests. These tests proved that the actuation time is interrupted by silver corrosion phenomena in screen-printing devices and by hot spots in inkjet-printing ones. Appropriate solutions regarding isolation and design improvements achieved not only an increase of lifetime and reliability, but also a decrease of power consumption.
\end{abstract}

Key words: Inkjet printing, screen printing, silver corrosion, flexible gas sensor.

\section{Introduction}

Global market demands for high quality and low-cost electronic devices. Printing techniques onto flexible polymeric substrates not only can lower the production costs but also offer the possibility to fabricate lightweight and wearable devices, unlike traditional electronic fabrication techniques such as monolithic silicon electronics using lithography and other patterning techniques [1-5].

Printed techniques have a high potential due to the possibility to work under room temperature and ambient pressure allowing a low-cost production [6]. SP (Screen-printing) is one of the main conventional and mature printing techniques, often used for simple industrial tasks (text printing and resist etch) and also complex tasks (printing conductors for flexible electronics and keypads). SP has demonstrated to be a useful technique in high-volume devices production; however, the need of masks makes the process

Corresponding author: Beatriz Medina-Rodriguez, Ph.D. student, research field: printed electronics. E-mail: b.medina@fae.es. tiresome for short series or customized products. For this reason, industrial companies search for new forms of printing techniques.

In the case of IP (Inkjet-printing), individual and micrometric drops are deposited onto the substrate on demand. The precise drop deposition opens the possibility of printing very thin conductive paths, minimizing both, device size and material expenses as well as the time and cost saving due to the ease in accomplishing design changes where the mask is not needed. IP is still under development and shows a huge challenge in terms of performance and reproducibility compared to matured techniques such as SP, and also in the current assortment of functional materials available on the market [8].

PET (Polyethylene terephthalate) and PEN (Polyethylene naphthalene) are both highly widespread as substrates in the current flexible electronic industry [9-14]. Nevertheless, PET and PEN have low thermal resistance and become unstable at temperature beyond $80{ }^{\circ} \mathrm{C}$ and $140{ }^{\circ} \mathrm{C}$, respectively, and lose their mechanical properties. Up these 
temperatures some others polymers, such as PI (Polyimide) are required for the post-treatment of the functional material $[13,15]$. PI can endure a widest temperature range. Its glass transition temperature is $360{ }^{\circ} \mathrm{C}$ what enhances its potential application in different technological fields.

Regarding printed materials; silver is the most widely used conductive material in flexible electronics. Its excellent electrical conductivity and the possibility to be sintered at low temperatures (lower than $300^{\circ} \mathrm{C}$ ) make it a good prospect for flexible devices. Even so, silver corrodes easily in industrial environments containing sulfide [17] or in outdoor applications due to the humidity [18] and chloride [19] even not near saltwater sources where there are inland chloride species, such as $\mathrm{ClNO}_{2}$ [20]. Therefore the reliability of the devices is highly compromised.

In this work, a comparison between low-cost flexible sensor platforms fabricated by means of SP and IP techniques has been developed. The performance of the sensor platforms was checked by long-term characterization and aging tests to identify the causes of the device failure. Design improvement to overcome the drawbacks of silver corrosion and power consumption has been achieved, obtaining a sturdy, wearable and reliable gas sensor platform.

\section{Experimental Setup}

\subsection{Techniques and Materials}

For the SP-devices a paste based on silver flakes (C2080415D2 from Gwent Group) was used as the conductive material to print the sensing and the heating parts of the gas sensor platform (Fig. 1a). Apical ${ }^{\circledR}$ PI from Sertek was used as the substrate and the SP process has been carried out with a DEK Horizon 03i. After printing, the silver paste was cured at $135{ }^{\circ} \mathrm{C}$ during $15 \mathrm{~min}$ in a box oven. These SP-devices were fabricated in FAE Company.

The IP-devices were printed with a Xenjet 4000 from Xennia Technology Ltd. The cartridge was a Xaar $126 / 50$, based on piezoresistive technology to eject the ink. U5603 silver ink with $20 \mathrm{wt} \%$ of silver nanoparticles was provided by Sun Chemical. In this case, Kapton ${ }^{\circledR}$ PI from DuPont was used as the substrate. These devices were fabricated in the Electronics Department of the University of Barcelona. The IP-patterns were sintered at $225^{\circ} \mathrm{C}$ for 20 min on a box oven. The adhesive PI tape used for the heater isolation was ISOAD 7104 provided by Isovolta.

The devices were calibrated and tested applying voltage pulses and monitoring their temperature by a thermographic camera (NEC Thermoshot F30). Long-term tests were carried out by applying a constant voltage to achieve an initial sensor temperature value of $120{ }^{\circ} \mathrm{C}$. Previous works done in our group showed that these platforms, implemented with carbon nanofibers as sensing material, better respond at $120{ }^{\circ} \mathrm{C}$ for $\mathrm{NH}_{3}$ and $\mathrm{NO}_{2}$ gas sensing [21]. The tests were interrupted different times to measure the heater resistance at room temperature. The chemical composition of the deposited materials was determined by EDXS (energy dispersive X-ray spectroscopy). The surface morphology of the deposited silver was checked by FE-SEM (field emission scanning electron microscopy). Both EDXS and FE-SEM analysis were carried out on a FE-SEM JEDL J-7100 microscope. Corrosion resistance of the deposits was analyzed by salt-spray chamber and by a

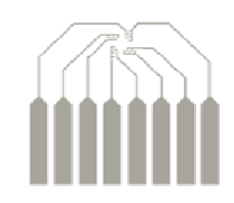

sensing part

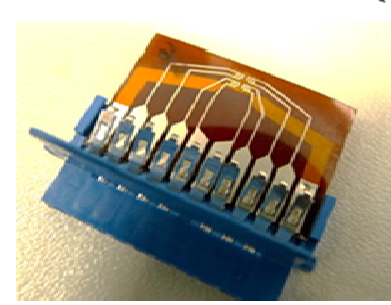

(b)

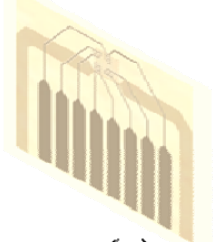

(a)
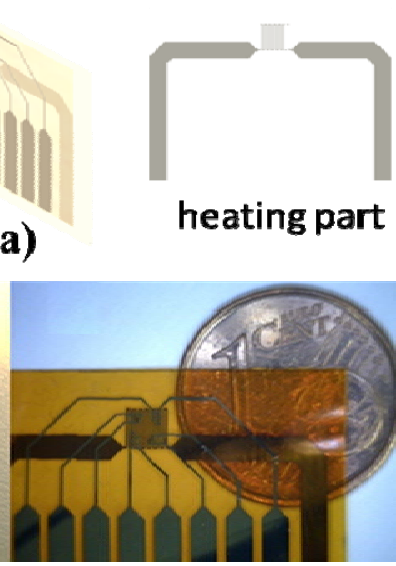

(c)

Fig. 1 (a) Scheme of the sensing and heating parts of the printed platforms, (b) SP-printed and (c) IP-printed 
devices.

climate chamber. Salt-spray tests were performed with a $50 \mathrm{~g} / \mathrm{L}$ of $\mathrm{NaCl}$ concentration during $72 \mathrm{~h}$ while climate chamber tests were performed with $95 \%$ humidity using two-hours on/off cycles keeping a temperature constant at $100^{\circ} \mathrm{C}$ during $72 \mathrm{~h}$.

\subsection{Design and Fabrication}

The sensor platform consists of a sensing part and a heater. The sensing part is composed by four interdigitated sensor electrodes and eight connection pads (Fig. 1a). The connection pads were designed in such a way that fit in a clamp-like connector, as it can be seen in Fig. 1b. While sensing part was printed in the topside of the PI tape, the heater was printed on the bottom side, being located just in the area where the printed electrodes are allowing heating up the sensing part. This kind of design (Fig. 1c) is already proved to be suitable for gas sensors [21]. The line width of the interdigitated electrodes and the line strip of the heater were $100 \mu \mathrm{m}$ and $150 \mu \mathrm{m}$, respectively.

\section{Experimental Results}

Once the devices were fabricated by means of SP and IP, heaters were calibrated. The sensing part temperature is plotted as a function of heater power consumption (Fig. 2). Both platforms showed similar behavior; the sensor temperature linearly increases as power consumption of the heater is higher, achieving around $120{ }^{\circ} \mathrm{C}$ for $200 \mathrm{~mW}$. This means that with SP and IP techniques, it is possible to fabricate devices with similar initial electrical characteristics.

To check the endurance of the heaters, a long-term test was carried out. It is known that silver oxidizes quicker as the temperature increases [22], so the heaters were connected to a voltage source to increase the sensor part temperature up to $120{ }^{\circ} \mathrm{C}$. Meanwhile, this temperature was kept constant; the printed devices were maintained in continuous operation until their failure. The heater electrical resistance was measured in different times of the test (square pattern line of Fig. 3a and b). After just few hours of operation,
SP-heaters evidenced a change on the surface color, from silvery to brown; this fact clearly indicates the presence of surface corrosion. A total device failure occurred after $300 \mathrm{~h}$ (Fig. 3a) where the electrical resistance of the heater increases asymptotically with time. Higher power consumption is needed for the same temperature, which being detrimental for the heater performance and leading to a malfunction of the devices. It is important to underline the fact that all the area which is heated up is degraded quickly; not only the heaters, but the sensing electrodes too. The EDXS analysis showed that only $\mathrm{Ag}$ and $\mathrm{C}$ elements were present in the as-deposited tracks (Table 1). After the SP-device failure, new elements as $\mathrm{S}, \mathrm{Cl}$ and $\mathrm{O}$ were found, which indicates a change of the track composition due to a surface corrosion. Furthermore, different studies [23-26] show that the porosity and roughness of metal coating surfaces accelerates the corrosion process. The FE-SEM images of SP-printings showed a no-homogenous, porous and full of holes surface, induced by the flake-like structure (Fig. 3c), which makes easier the penetration of the reactive species, like $\mathrm{S}, \mathrm{Cl}$ and $\mathrm{O}$, into the deposit structure, causing then the premature failure.

Regarding to IP-devices, the total failure occurred in $140 \mathrm{~h}$ (square pattern line Fig. 3b), earlier than SP-devices, but no surface corrosion evidences were found. EDXS analysis showed no significant variation on the deposit composition of IP-devices after the

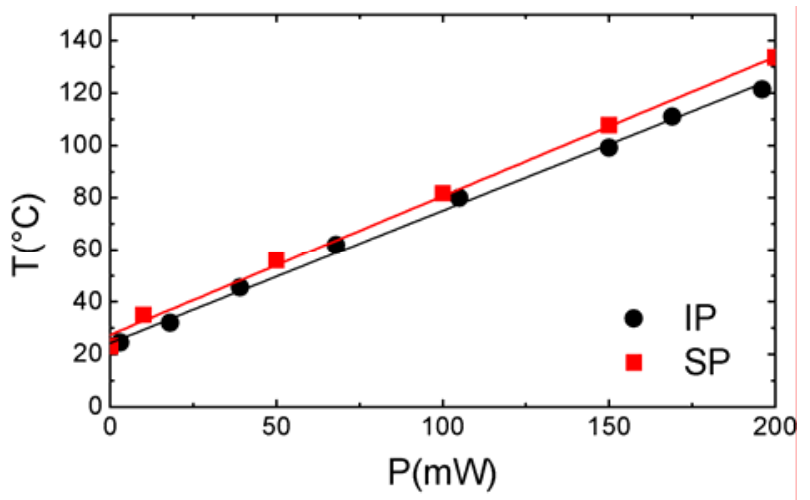

Fig. 2 Calibration plot of SP- and IP-devices. A linear dependence of the temperature of the sensing part is evidenced as a function of heater power consumption. 
Screen printing devices

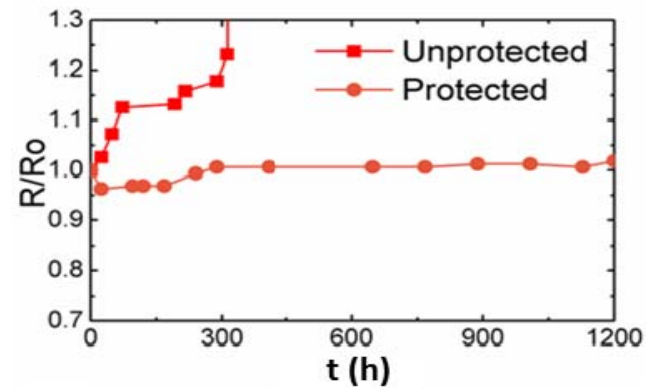

(a)

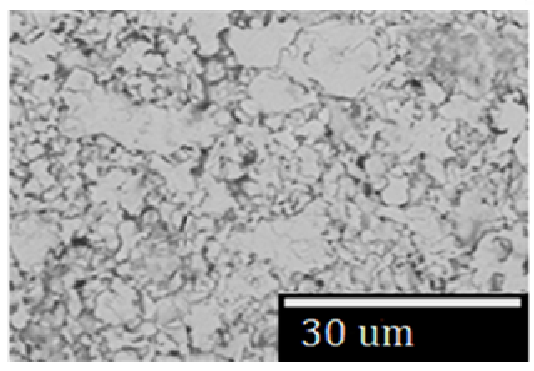

(c)
Inkjet printing devices

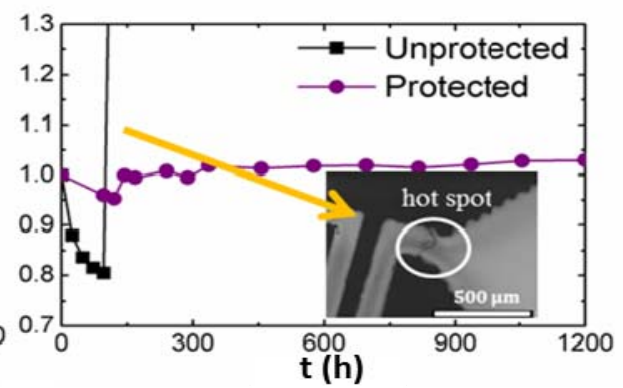

(b)

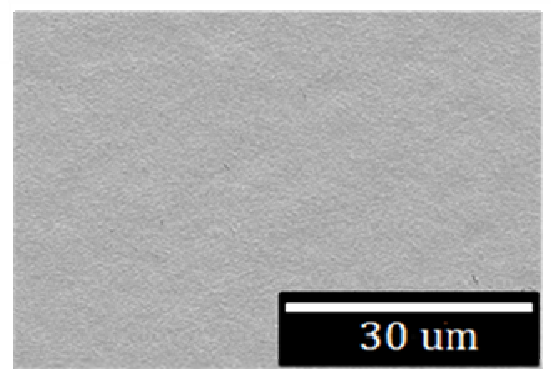

(d)

Fig. 3 Electrical performances of (a) SP- and (b) IP- heaters during the continuous operation at $120{ }^{\circ} \mathrm{C}$. FE-SEM images of the silver track printed by (c) screen printing and (d) inkjet printing. Inset: discontinuity on the inkjet-printed heater caused by a hot spot.

Table 1 EDX results for the composition of the printed deposits before and after the long-term test.

\begin{tabular}{lllll}
\hline \multirow{2}{*}{ Element } & \multicolumn{2}{c}{ SP devices (wt. \%) } & \multicolumn{2}{c}{ IP devices (wt. \%) } \\
\cline { 2 - 5 } & Before & After & Before & After \\
\hline $\mathrm{Ag}$ & 92 & 79 & 98 & 95 \\
$\mathrm{C}$ & 8 & 8 & 2 & 3 \\
$\mathrm{~S}$ & 0 & 6 & 0 & 0 \\
$\mathrm{Cl}$ & 0 & 3 & 0 & 0 \\
$\mathrm{O}$ & 0 & 4 & 0 & 2 \\
\hline
\end{tabular}

failure (Table 1). FE-SEM images (Fig. 3d) showed a homogeneous surface without the presence of holes or defects. A completely smooth surface is reached by means of the sintering process of the silver nanoparticles. IP-devices showed then an almost inexistent porosity that minimizes the silver exposed to the atmosphere, slowing down the penetration of reactive elements and so the corrosion. Thereby, corrosion is not the cause of these IP-devices failure.

A detailed examination revealed the presence of some discontinuities on the silver tracks after the long-term tests. These discontinuities appeared at vulnerable and weak parts of the design (Fig. 3b) or at printing defects (bubbles, impurities). Unlike the SP-devices, where the single layer track thickness was around $20 \mu \mathrm{m}$, the single printed layer of the IP-tracks was $300 \mathrm{~nm}$ thick, what might promote the formation and the fast evolution of cracks and hot spots in a short period of time.

The aging tests showed more obvious the penetration of the reactive species into the silver deposit structure. The SP-heater resistance was increased around $7.6 \%$ and $10 \%$ after the climate and the salt-spray chambers exposure, respectively. However, for IP-devices the increase of the resistance was quite low; $1.3 \%$ and $2.7 \%$ for climate and spay-salt chambers, respectively, lower than SP-devices (Table 2).

With the aim to improve the life-time of the designed platforms and to prevent the premature failure of both, SP- and IP-devices, an atmospheric isolation of the silver heaters by a suitable adhesive PI tape was carried out. The sensing part has to be exposed to atmosphere to work as a gas sensor device, 
thereby it cannot be isolated. It has to be noticed that after the device protection, devices were laminated to remove all the air that could be trapped between the two layers. By means of the heater protection, long-term test showed an increase of the lifetime of protected SP- and IP-devices more than ten times compared with the un-protected ones (circle pattern line in Fig. 3a and Fig 3b). No degradation after $1,000 \mathrm{~h}$ of continuous operation at $120{ }^{\circ} \mathrm{C}$ and no consequential deterioration on the device performance were observed. However, the SP-electrodes of the sensing part, which could not be protected from the atmosphere, corroded in few hours due to the porous presence, as it was previously mentioned. Instead, the IP-devices showed no corrosion on the electrodes after $1,000 \mathrm{~h}$ of continuous operation. Furthermore, it has been demonstrated that heater protection not only improves SP- but also the IP-devices. The aging tests carried out on the protected devices revealed smaller increase of electrical resistance than for unprotected-devices. An increase around $0.6 \%$ and $2 \%$ was observed in the case of SP-devices for climatic and salt-spray chambers, respectively. No significant variation in electrical resistance of IP-devices was measured. Then, long-term reliability was achieved by means of the heater protection for both SP- and IP-devices, just the unprotected electrodes made the difference.

At this point, it seems that IP-technique can be pointed as the best route to fabricate such flexible sensing platforms. For this reason, IP heater design was optimized to achieve lower power consumption. As said previously, the very first devices showed a power consumption of almost $200 \mathrm{~mW}$ at a temperature of $120{ }^{\circ} \mathrm{C}$ (Fig. 2). For new design, the heated area was reduced by narrowing the strips as it is shown in Fig. 4a. Once these changes were carried out, the power consumption was reduced to $125 \mathrm{~mW}$ at $120{ }^{\circ} \mathrm{C}$ for protected inkjet-printed heaters (Fig. 4b) which implies a reduction of a $38 \%$ on power consumption, which value also correspond to the heating area reduction.

Silicon technology is energetically a little more efficient, around few tens of $\mathrm{mW}$ for $200{ }^{\circ} \mathrm{C}[27,28]$, but the fabrication process is extremely expensive and time consuming. An alternative can be the ceramic technology because it is cheaper, but in this case the power consumption increases excessively on the order of hundred times more [29, 30], as well than on glass-based devices [31]. Therefore, the methodology here proposed can be considered like a good alternative to both technologies, reaching a low-consumption and low-cost sensor platform.

Additionally, it has to mention that this novel flexible-platform was implemented with deposited carbon-based materials as the gas sensing material, by means of electrospray technique. The success of this system has been proved in Ref. [21] by measuring different gases such as $\mathrm{NH}_{3}$ or $\mathrm{NO}_{2}$.

Table 2 Variation of the electrical resistance for the different devices after the aging test.

\begin{tabular}{lll}
\hline & $\begin{array}{l}\text { Salt-spray chamber } \\
\text { test } \Delta \text { R/Ro }(\%)\end{array}$ & $\begin{array}{l}\text { Climate chamber test } \\
\Delta \mathrm{R} / \text { Ro }(\%)\end{array}$ \\
\hline SP & 10.0 & 7.6 \\
Protected SP & 2.0 & 0.6 \\
IP & 2.7 & 1.3 \\
Protected IP & 0.1 & 0.1 \\
\hline
\end{tabular}

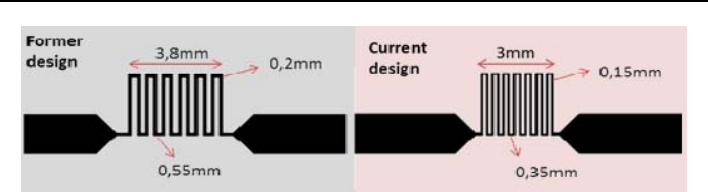

(a)

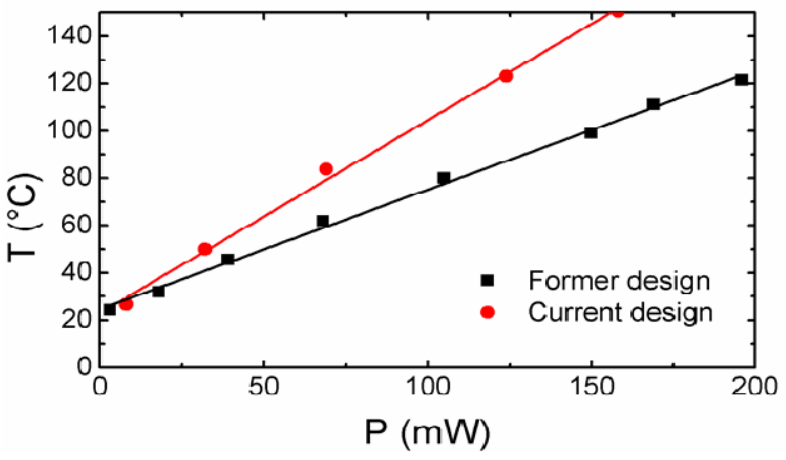

(b)

Fig. 4 (a) Scheme and dimensions of the heaters; (b) Sensor temperature as a function of the heater power consumption. 


\section{Conclusions}

Flexible gas sensor platforms fabricated by means of screen- and inkjet-printing were tested by long-term tests maintaining $120^{\circ} \mathrm{C}$ during heater operation. Early device failure, caused by chemical degradation, is observed in SP-devices due to the flake-like morphology of the deposits. No noticeable deterioration occurs in IP-conductive tracks of silver nanoparticles. However, the very thin film obtained by inkjet printing promotes a failure by hot spot phenomena. Both kinds of failure are prevented by the heaters isolation from the atmosphere by an adhesive PI tape. The SP- and IP-devices lengthen their life time more than 10 times the unprotected ones. The homogeneity and the smooth surface of IP-devices make them being operative more time than SP-ones. For IP-devices, an improvement of the heater design by reducing the exposed area has shown a decrease of power consumption of $38 \%$, what position the sensor platform as a low-cost and low-powered consumer device. It has been demonstrated that the synergetic combination of polymeric substrates with printed electronics is a good alternative to substitute the monolithic silicon and the ceramic technologies.

\section{Acknowledgments}

Albert Cirera acknowledges financial support from ICREA Academia program. Beatriz Medina-Rodriguez acknowledges financial support from Doctorat Industrial program (ACCIÓ-Generalitat de Catalunya).

\section{References}

[1] Zardetto, V., Brown, T. M., Reale, A. and Di Carlo, A. 2011. "Substrates for Flexible Electronics: A Practical Investigation on the Electrical, Film Flexibility, Optical, Temperature, and Solvent Resistance Properties.” Journal of Polymer Science Part B: Polymer Physics 49: 638-48.

[2] MacDonald, W. A., Looney, M. K., MacKerron, D., Evesn, R., Adam, R. and Hashimoto, K. et al. 2007. "Latest Advances in Substrates for Flexible Electronics." Journal of the SID 15 (12): 1075-83.

[3] Bao, Z. 2000. "Materials and Fabrication Needs for
Low-Cost Organic Transistor Circuits." Advanced Materials 12 (3): 227-30.

[4] Sankır, N. D. 2005. "Flexible Electronics: Materials and Device Fabrication." Ph.D. Thesis, Virginia Polytechnic Institute and State University in Blacksburg.

[5] Menard, E., Meitl, M. A., Sun, Y., Park, J. U., Shir, D. J. L. and Nam, Y. S. et al. 2007. "Micro-and Nanopatterning Techniques for Organic Electronic and Optoelectronic Systems.” Chemical Reviews 107 (4): 1117-60.

[6] Kebs, F. C. 2009. "Fabrication and Processing of Polymer Solar Cells: A Review of Printing and Coating Techniques." Solar Energy Materials \& Solar Cells 93 (4): 394-412.

[7] Perelaer, J. and Schubert, U. S. 2010. "Inkjet Printing and Alternative Sintering of Narrow Conductive Tracks on Flexible Substrates for Plastic Electronic Applications, Radio Frequency Identification Fundamentals and Applications, Design Methods and Solutions." In InTech, edited by Turcu, C., 324.

[8] Hudd, A. 2010. "The Chemistry of Inkjet Inks.” in: (Ed.), WorldScientific, New Jersey-London-Singapore, edited by Magdassi, S.3-18.

[9] Sun, Y., and Rogers, J. A. 2007. "Inorganic Semiconductors for Flexible Electronics." Advanced Materials 19: 1897-916.

[10] Sazonov, A., Striankhilev, D., Lee, C.-H., and Nathan, A. 2005. "Low-Temperature Materials and Thin Film Transistors for Flexible Electronics." In Proceedings of the IEEE 93 (8): 1420-8.

[11] Hu, A., Guo, J. Y., Alarifi, H., Patane, G., Zhou, Y. and Compagnini, G. et al. 2010. "Low Temperature Sintering of Ag Nanoparticles for Flexible Electronics Packaging." Applied Physics Letters 93: 153117.1-7.3.

[12] Jahn, S. F., Blaudeck, T., Baumann, R. R., Jakob, A., Ecorchard, P. and Rüffer, T. et al. 2010. "Inkjet Printing of Conductive Silver Patterns by Using the First Aqueous Particle-Free MOD Ink without Additional Stabilizing Ligands." Chemistry of Materials 22 (10): 3067-71.

[13] Kim, D., and Moon, J. 2005. "Highly Conductive Ink Jet Printed Films of Nanosilver Particles for Printable Electronics." Electrochemical and Solid-State Letters 8 (11): 30-3.

[14] Magdassi, S., Grouchko, M., Berezin, O. and Kamyshny, A. 2010. "Triggering the Sintering of Silver Nanoparticles at Room Temperature." ACS Nano 4 (4): 1943-8.

[15] Lee, Y. I., and Choa, Y. H. 2012. "Adhesion Enhancement of Ink-Jet Printed Conductive Copper Patterns on a Flexible Substrate." Journal of Materials Chemistry 22 (25): 12517-22.

[16] Scandurra, A., Indelli, G. F., Spartà, N. G., Galliano, F., 
Ravesi, S. and Pignataro, S. 2010. "Low-Temperature Sintered Conductive Silver Patterns Obtained by Inkjet Printing for Plastic Electronics." Surface and Interface Analysis 42 (6-7): 1163-7.

[17] Minzari, D., Jellesen, M. S., Moller, P. and Ambat, R. 2011. "Morphological Study of Silver Corrosion in Highly Aggressive Sulfur Environment." Engineering Failure Analysis 18 (8): 2126-36.

[18] Lin, H., and Frankel, G. S. 2013. "Accelerated Atmospheric Corrosion Testing of Ag." Corrosion 69 (11): 1060-72.

[19] Liang, D., Allen, H. C., Frankel, G. S., Chen, Z. Y., Kelly, R.G. and Wu, Y. et al. 2010. "Effects of Sodium Chloride Particles, Ozone, UV, and Relative Humidity on Atmospheric Corrosion of Silver." Journal of Electrochemical Society 157: 146-56.

[20] Lemon, C. E. 2012. "Atmospheric Corrosion of Silver Investigated by X-ray Photoelectron Spectroscopy.” Ph.D. Thesis, The Ohio State University.

[21] Claramunt, S., Monereo, O., Boix, M., Leghrib, R., Prades, J. D. and Cornet, A. et al. 2013. "Flexible Gas Sensor Array with an Embedded Heater Based on Metal Decorated Carbon Nanofibres." Sensors and Actuators B: Chemical 187: 401-6.

[22] Sakai, J., Aoki, L., Ohsaka, K. and Ishikawa, Y. 2005. "Sub-micrometer Order Corrosion of Silver by Sulfur Vapor in Air Studied by Means of Quartz Crystal Microbalance." In International Corrosion Congress, 19-24.

[23] Varea, A., Pellicer, E., Pané, S., Nelson, B. J., Suriñach, S. and Baró, M. D. et al. 2012. "Mechanical Properties and Corrosion Behaviour of Nanostructured $\mathrm{Cu}$-rich CuNi Electrosdeposited Films." International Journal of Electrochemical Science 7: 1288-302.

[24] Bersirova, O., Kublanovsky, V., Anufryiev, L. and
Rubtsevich, I. 2004. "Corrosion Behavior of Electroplated Silver Coatings." Materials Science 10 (1): $11-4$.

[25] Bersirova, O. L. and Kublanovskii, V. S. 2012. "Corrosion Properties of Electrodeposited Thin Coatings of Polycrystalline Silver.” Materials Science 48 (2): 197-202.

[26] Kaushik, V. K. 1991. "XPS Core Level Spectra and Auger Parameters for Some Silver Compounds." Journal of Electron Spectroscopy and Related Phenomena 56 (3): 273-7.

[27] Cerdà, J., Cirera, A., Vilà, A., Cornet, A. and Morante, J. R. 2001. "Deposition on Micromachined Silicon Substrates of Gas Sensitive Layers Obtained by a Wet Chemical Route: a $\mathrm{CO} / \mathrm{CH}_{4}$ High Performance Sensor." Thin Solid Films 391 (2): 265-9.

[28] Vilaseca, M., Coronas, J., Cirera, A., Cornet, A., Morante, J. R. and Santamaria, J. 2008. "Development and Application of Micromachined $\mathrm{Pd} / \mathrm{SnO}_{2}$ Gas Sensors with Zeolite Coatings." Sensors and Actuators B: Chemical 133 (2): 435-41.

[29] Moldavan, C., Nedelcu, O., Johander, P., Goenaga, I., Gomez, D. and Petkov, P. et al. 2007. "Ceramic Micro Heater Technology for Gas Sensors.” Romanian Journal of Information Science and Technology 10 (1): 43-52.

[30] Jiang, B., Maeder, T., Santis-Alvarez, A. J., Poulikakos, D. and Muralt, P. 2014. "A Low-Temperature Co-Fired Ceramic Micro-Reactor System for High-Efficiency On-Site Hydrogen Production." Journal of Power Sources 273: 1202-17.

[31] Santis-Alvarez, A. J., Nabavi, M., Jiang, B., Maeder, T., Muralt, P. and Poulikakos, D. 2012. "A Nanoparticle Bed Micro-Reactor with High Syngas Yield for Moderate Temperature Micro-Scale SOFC Power Plants." Chemical Engineering Science 84: 469-78. 